\title{
Somatosensory amplification, depression, anxiety and pain in statin intolerant patients
}

\author{
Jasleen Singh $^{1}$, Sara Sirna ${ }^{2}$, Khaled Dajani ${ }^{2}$, Shermeen Memon ${ }^{1}$ and Angelos Halaris ${ }^{2 *}$ \\ ${ }^{1}$ Department of Psychiatry, Loyola University Medical Center, 2160 S 1st Ave, Maywood, IL 60153, USA \\ ${ }^{2}$ Division of Cardiology, Department of Medicine, Loyola University Medical Center, 2160 S 1st Ave, Maywood, IL 60153, USA
}

\begin{abstract}
Background: Lipid management with statins is one of the mainstays of treatment for atherosclerotic cardiovascular disease. Statins are often discontinued by patients secondary to muscle-related complaints independent of elevations in creatine kinase. It has also been suggested that there is a link between mental health disorders and noncompliance. The associations between negative affect and somatization reported as medication-related side effects requires further clarification. One possible cause of statin discontinuation is perceived myopathy secondary to somatization.

Objective: This study examines the relationship between affect in statin tolerant and statin intolerant (SI) groups.

Methods: This study incorporated patients unable to tolerate at least two different statins (any statin or dosage prescribed) due to side effects or abnormal laboratory markers. Patients completed The Depression Anxiety Stress Scales, the McGill Pain Questionnaire, and the Somatosensory Amplification Scale.

Results: There were 20 statin tolerant (ST) patients and 13 SI patients ( $\mathrm{n}=36)$. There was a significant difference between SI and ST groups, respectively, in age (63 vs. 70 years, $\mathrm{p}=0.04)$, total cholesterol (204 vs.136, p <0.01), and LDL cholesterol (129 vs. 136, p < 0.05). There were no significant differences between ST and SI groups for somatic pain, affective pain, somatosensory amplification, depressive symptoms, anxiety symptoms, and stress scores.

Conclusion: Our study suggests the discrepancy between statin intolerance in clinical trials and clinical practice cannot be explained solely by somatosensory amplification, anxiety, depression or pain disorders. Nevertheless, an affective and/or anxiety disorder will likely augment the pain perception and somatization in a SI patient.
\end{abstract}

\section{Introduction}

Cardiovascular disease (CVD) is the leading global cause of death, accounting for 17.3 million deaths per year and expected to reach 23.3 million deaths per year by 2030 [1,2]. Many modifiable risk factors are linked to CVD, including hyperlipidemia, which affects an estimated 53\% of United States adults [3]. Given the high prevalence of dyslipidemia and its association with CVD, statins are the cornerstone therapy for reducing risk of atherosclerostic CVD via lipid management. Clinically, statins produce a quantifiable reduction of LDL-C ( 30-60\%) and stabilize atherosclerotic plaques, reduce inflammation, and reduce endothelial dysfunction. Yet, randomized controlled trials identified safety concerns in persons on statins. Thus, physicians should consider patient age, cardiovascular risk, and comorbidities such as impaired renal or hepatic function, history of previous statin intolerance or unexplained elevated transaminase greater than three times the upper limit of normal or creatinine kinase (CK), that predispose patients to adverse effects from statins. Statins are often discontinued due to intolerance, manifested by muscle pain or weakness independent of an elevated CK, and an estimated $29.9 \%$ of patients stop their statin therapy in the first year [4,5]. The Heart Protection Study found that one-third of middle-aged or elderly patients reported muscle aches or weakness at least once while on a statin [6]. Interestingly, psychosomatic symptoms, such as muscle aches and pain can be associated with stress, depression, and other psychological impairments common in CVD patients [7-10]. A meta-analysis found that depressed patients were three times more likely to be noncompliant with medical treatment recommendations
[11]. Thus, we sought to study the relationship between negative affect and somatic symptoms, suggesting that if statin intolerance is partially due to somatization, we would see differences in affect between statin tolerant and statin intolerant groups.

\section{Methods}

\section{Design}

The study was approved by the Institutional Review Board of Loyola University Medical Center and all participants provided written informed consent. We employed a one-time quantitative assessment to explore the clinical presentations of statin intolerant (SI) patients and analyze the differences in affective, somatic, and physical symptoms between SI and statin tolerant (ST) patients.

\section{Subjects}

Patients enrolled in this study received care as outpatients in the Cardiology Division at Loyola University Medical Center. Inclusion

${ }^{\star}$ Correspondence to: Angelos Halaris, Division of Cardiology, Department of Medicine, Loyola University Medical Center, 2160 S 1st Ave, Maywood, IL 60153, USA, E-Mail: ahalaris@lumc.edu

Key words: myopathy, negative affect, somatization, somatic symptoms, statin intolerance

Received: April 04, 2019; Accepted: April 15, 2019; Published: April 18, 2019 
criteria included patients over the age of 18 , previously diagnosed with SI by their medical providers, were able to provide informed consent, and read and write in English. Statin intolerance was defined when a patient was unable to tolerate at least two different statins due to the development of side effects or abnormal laboratory markers. Our study was limited to patients with complete statin intolerance, unable to tolerate any statin or dosage prescribed. Moreover, patients were excluded if they were currently receiving psychiatric treatment, such as psychotropic medications or psychotherapy, were cognitively or medically unable to complete study measures, or had a history of elevated CK levels and/or elevated LFT levels over 3 times the upper limit of normal.

\section{Study protocol}

Clinicians identified patients who presented to the Outpatient Cardiology Clinic at Loyola University Medical Center and who had been previously prescribed a statin. Patients were invited to participate if they met the above study inclusion and exclusion criteria and agreed. After obtaining informed consent, patients completed three surveys: The Depression Anxiety Stress Scales (DASS), the McGill Pain Questionnaire, and the Somatosensory Amplification Scale (SAS). After completion of the surveys, a chart review of each subject was performed assessing for other variables including a history of myocardial infarction, hypertension, diabetes mellitus, psychiatric history, statin medication history, and levels of total cholesterol, LDL, HDL, triglycerides and ALT.

\section{Rating scales}

The Depression Anxiety Stress Scales (DASS): The DASS-21 is a 21 item self-report questionnaire that is a dimensional rather than categorical assessment of psychological disorders. The 21 questions assess depression, anxiety, and stress focusing on negative emotional symptoms that have occurred over the past week with frequency and severity rated on a scale between 0 and 3. Each question separately analyzes depressive versus anxiety versus stress symptoms. The scaled scores for the depressive, anxiety and stress questions are summed separately and multiplied by two, allowing for comparison to the normative data for the original 42 -item scale. The scale is applicable to patient populations who are able to cognitively understand the questions and is not dependent on prior medical or psychiatric history $[12,13]$.

The Somatosensory Amplification Scale (SAS): The SAS is a 10item self-report questionnaire that measures how patients experience physical symptoms and assesses propensity for somatization. Each question is scored on a 5-point scale, and the scores are summed to 1050 , with higher scores having higher predictive values for somatization. The scale is applicable to the general community, patients with medical disorders, or patients with psychiatric disorders $[9,12,14]$.

The Short-Form McGill Pain Questionnaire: The Short-From McGill Pain Questionnaire (SF-MPQ) contains four different sections, with each section assessing different aspects of physical pain. The first section is a 15-question self-report questionnaire assessing pain quality via a Likert scale of intensity ( $0=$ none, $3=$ severe), and distinguishes sensory and affective pain. The following three sections assess intensity and the time course of pain using a visual analog scale (1-10), a present pain inventory scale ( $0=$ no pain, $5=$ excruciating), and a pattern of pain scale ( $1=$ brief, $2=$ intermittent, $3=$ continuous). The SF-MPQ highly correlates to the original McGill Pain Questionnaire and is applicable to any patient population [15].

\section{Statistical analysis}

IBM SPSS version 22 was used for statistical analyses. Mean differences in demographic information (age, BMI, race, gender) and clinical characteristics (total cholesterol, history of cardiac events, hypertension, diabetes, insomnia, depressive disorder and pain disorder) were determined using descriptive frequency statistics. Mean differences in age, BMI, total cholesterol, LDL, HDL, and ALT among the SI and the ST groups were analyzed by an independent samples t-test. Clinical outcome measures in the statin intolerant and statin tolerant patients were analyzed at the time they were consented. Distributions for all rating scales were scrutinized for normality and skewedness prior to the following analyses. Short-Form McGill Pain Questionnaire, Somatosensory Amplification Scale (SAS), and Depression, Anxiety, Stress Scale (DASS) scores were weighted and summed appropriately according to the scale reference. The scores were then analyzed by an independent samples t-test for normally distributed variables. A Pearson Correlation Coefficient analysis was performed with the statin intolerant group to determine if the number of statins a patient was determined to be intolerant to correlated to the rating scales used in the study.

\section{Results}

A total of 36 patients met study criteria and were enrolled after signing informed consent. All 36 completed the McGill Pain Questionnaire, Somatosensory Amplification Scale, and Depression Anxiety Stress Scale. Tables 1 and 2 show the demographic and clinical characteristics of the cohort of patients. Overall there were 20 ST patients and 13 SI patients. There was a significant difference between SI and ST groups, respectively, in age (63 vs. 70 years, $\mathrm{p}=0.04)$, total cholesterol (204 vs.136, p <0.01), and LDL cholesterol (129 vs. 136, p < 0.05) (Table 3 ). Table 3 shows the average scores of the DASS, SAS and SF-MPQ for ST and SI groups. There were no significant differences between ST and SI groups for somatic pain, affective pain, somatosensory amplification, depressive symptoms, anxiety symptoms, and stress scores.

\section{Discussion}

We hypothesized that patients who experienced statin intolerance would be more likely to express symptoms of a somatoform disorder,

Table 1. Demographics of subjects

\begin{tabular}{|c|c|}
\hline Demographics & $\begin{array}{c}\text { Subjects currently or previously taking } \\
\text { statin medication N=36 }\end{array}$ \\
\hline Age (SD) & $67.8(10.7)$ \\
\hline BMI (SD) & $30.3(6.1)$ \\
\hline Males (\%) & $19(52.8 \%)$ \\
\hline Females (\%) & $17(47.2 \%)$ \\
\hline Caucasian (\%) & $23(63.9 \%)$ \\
\hline African American (\%) & $7(19.4 \%)$ \\
\hline Hispanic (\%) & $2(5.6 \%)$ \\
\hline Asian (\%) & $1(2.8)$ \\
\hline Other (\%) & $3(8.3 \%)$ \\
\hline Non-Caucasian & $13(36.1 \%)$ \\
\hline
\end{tabular}

Table 2. Clinical characteristics of subjects

\begin{tabular}{|c|c|}
\hline Clinical Characteristic & $\mathrm{N}=36(\%)$ \\
\hline History of Cardiac Events & $13(36.1 \%)$ \\
\hline Hypertension & $26(72.2 \%)$ \\
\hline Type II Diabetes Mellitus & $15(41.7 \%)$ \\
\hline History of Insomnia & $6(16.7 \%)$ \\
\hline History of Depressive Disorder & $3(8.3 \%)$ \\
\hline History of Pain Disorder & $9(25.0 \%)$ \\
\hline
\end{tabular}


Table 3. Differences in rating scale scores between statin intolerant and statin tolerant groups (Note: $\mathrm{ST}=$ Statin Tolerant. SI $=$ Statin Intolerant)

\begin{tabular}{|c|c|c|c|}
\hline \multirow{2}{*}{ Rating Scale } & \multicolumn{2}{|c|}{ Mean (SD) } & \multirow{2}{*}{$\begin{array}{l}\text { Significance } \\
\text { p-value }\end{array}$} \\
\hline & ST $N=20$ & SI $N=13$ & \\
\hline McGill - Somatic & \multicolumn{2}{|c|}{$2.25(6.6)$} & $6.46(7.1)$ \\
\hline McGill - Affective & \multicolumn{2}{|c|}{$0.45(1.3)$} & $1.15(1.6)$ \\
\hline SAS & \multicolumn{2}{|c|}{$24.0(7.3)$} & $25.8(7.7)$ \\
\hline DASS- Depression & \multicolumn{2}{|c|}{$1.25(1.3)$} & $2.07(4.3)$ \\
\hline DASS- Anxiety & \multicolumn{2}{|c|}{$2.60(2.6)$} & $2.46(3.2)$ \\
\hline DASS- Stress & \multicolumn{2}{|c|}{$3.60(3.5)$} & $3.69(5.2)$ \\
\hline
\end{tabular}

as the number of somatic symptoms throughout one's lifetime has been shown to be independently and directly associated with the number of anxiety and depressive episodes [16-18]. Our study suggests that SI patients are no more likely to express symptoms of a somatoform disorder compared to ST patients. Based on the McGill-Somatic, McGillAffective, SAS, DASS-Depression, DASS-Anxiety, and DASS-Stress rating scales, there was no significant difference in scale scores between SI and ST patients. Despite no significant differences between ST and SI groups for somatic pain, affective pain, somatosensory amplification, depressive symptoms, anxiety symptoms and stress scores, we noted many of the patients who were SI and excluded from the study were already prescribed anti-anxiety and anti-depressant medications. If these patients deemed SI were enrolled in the study, a difference may have been observed; however, this would have introduced significant confounders.

Studies have suggested that psychiatric diagnoses such as anxiety and depression, in the context of a chronic medical illness, lead to increased physical manifestations of the disease compared to medical illness alone, increased awareness of symptoms, and increased impression of symptom burden $[10,18,19]$. There is also evidence showing that improvement in depression in the setting of chronic medical illness leads to decreased symptom burden [16]. While the relationship between somatization, the prevalence of negative affective symptoms, and alexithymia have not been shown in relation to myopathy or statin intolerance, clinical indications show that patients with SI tend to have more distress, and difficulty with pain perceptions compared to ST patients. Furthermore, alexithymia, or the tendency to focus on external symptoms secondary to an inability to recognize emotional symptoms in oneself, is highly associated with anxiety sensitivity, somatosensory amplification and effects the way patients seek and receive care $[10,12,20-22]$.

Of note, patients with somatoform disorders have higher levels of salivary cortisol, increased heart rate, and lower digital pulse volume while under stress [23]. This indicates that perceived external stimuli lead to different physiologic responses [24]. This is supported by a prospective cohort study of over 1,900 patients that found an association between statin non-adherence and somatic symptoms of anxiety, suggesting that these symptoms were often misinterpreted as being a result of the medication instead of a manifestation of their anxiety [25]. A related phenomenon that emphasizes the impact of perception on physical symptomatology and has been reported in the literature is the nocebo effect, which is when the patient's expectation that there will be an adverse effect results in increased rates of negative outcomes. In fact, a 2017 study demonstrated a direct correlation between the prevalence of SI and the number of websites about statin side effects that a patient could find via Google search [26]. The nocebo effect is a result of neuropsychology rather than representative of a drug's efficacy or toxicity, yet the important distinction is that this effect is a somatic manifestation secondary to a patient's preconceived misperceptions. This is supported by randomized controlled trials that have demonstrated no significant difference in the incidence of muscle complaints and overall rate of statin discontinuation between patients receiving statins and placebos [27,28]. Furthermore, randomized controlled trials that re-challenged patients deemed SI have also demonstrated muscle symptoms related to the nocebo effect, while acknowledging there are also a subset of patients who experience these symptoms as a pharmacological effect of the statin itself. As a result, it is likely that multiple factors may play a role in this phenomenon, including cultural and societal factors relating to the stigma around psychological distress coupled with an acceptance towards physical complaints. Thus, one way to minimize this effect includes improvements in patient education and patient-provider communication [27].

While our study suggests that SI patients are no more likely to express symptoms of a somatoform disorder compared to ST patients, it remains plausible that myopathy in the presence of statin therapy may be at least partially explained by the presence of a somatoform disorder. Our study is the first to examine somatic tendencies or negative affective differences between SI and ST patients. Given the sample size limitation of our study, a much larger study evaluating the relationship between negative affective syndromes and statin intolerance should be performed.

There is little doubt that adverse effects of statins affect many patients, especially middle-aged and elderly women. Fortunately, many of these patients can tolerate either a lower dose statin or a different statin without limiting side effects. We hypothesized that statin intolerance, especially when related to myopathy, is multifactorial and may still involve the presence of a psychological disorder and stressors. One significant limitation of our study involved the extreme difficulty in enrolling SI patients who were not taking prescribed anxiolytics or antidepressants by their primary care providers. Referral to a mental health professional may be helpful in some cases to mediate statin side effects and/or relieve some anxiety related to heart disease and drug intolerance.

We are therefore more inclined to attribute SI manifested predominantly in myopathy and myositis as a biological rather than solely a psychological phenomenon. Proposed biological mechanisms of myopathic SI include genetic predisposition and increased bioavailability. Multiple etiologies of statin-induced myopathy have been identified, including metabolic changes due to CYP enzyme variations, altered pharmacokinetic profiles secondary to variations in $\mathrm{ABC}$ transporter genes, diagnosis with a metabolic myopathy, decrease in farnesyl pyrophosphate production, impairments in calcium signalling, mitochondrial dysfunction in association with decreased levels of Coenzyme Q10, decreased protein synthesis, myalgias secondary to Vitamin D deficiency, and alterations in protein degradation $[29,30]$. A study investigating genetic variants of LILRB5, a leukocyte immunoglobulin-like receptor subfamily-B that has been associated with decreased levels of creatine phosphokinase and lactate dehydrogenase found that homozygotes of the Asp247 variant had increased risk of myalgia, yet carriers of the $247 G l y$ variant were found to have an increased risk of statin-induced myalgia [31].

The observation that SI disappears in some patients after switching to a different statin supports the argument that the primary cause of SI should be sought in inflammatory and/or immune processes. Autoimmune myositis has also been described in the literature as a possible contributory cause to SI. Notably, a progressive and persistent form of statin-associated immune-mediated necrotizing myopathy 
(IMNM) has been identified in association with anti-5-hydroxy-3methylglutaryl coenzyme A reductase (HMGCR) autoantibodies, with an estimated annual incidence of two per million and prevalence of one per 100,000. Diagnosis of SI secondary to this etiology may be assisted via muscle biopsy demonstrating necrosis. These patients typically present with severe symptoms requiring immunosuppressive treatment [30]. One study found an increased prevalence of antiHMGCR autoantibodies in statin users compared to non-users in patients with idiopathic inflammatory myopathies as well as a subset of patients with IMNM, with these patients also demonstrating higher titers of these antibodies and more commonly having muscle necrosis [32]. Interestingly, anti-HMGCR antibodies have been detected in patients who have never taken statins, as well. One hypothesis for this phenomenon is the presence of natural statins in many foods, yet it was also noted that these patients tended to be younger age, of African American descent, and had higher levels of creatine kinase $[32,33]$.

Given the symptom burden of IMNM, it may be worth considering testing for anti-HMGCR antibodies via immunoassays in statin-exposed patients with clinical suspicion for this disease process. In addition to testing for these antibodies via enzyme-linked immunosorbent assay (ELISA) or chemiluminescence immunoassay (CIA), one study also identified a distinct indirect immunofluorescence (IFL) staining pattern, HMGCR associated liver IFL pattern or HALIP, for HMGCR-associated myopathy [34]. Of course, cost-effectiveness of such a test must also be taken into consideration. This was addressed by a study that examined the economic feasibility of a theoretical pharmacogenomic test for SI secondary to myopathy in high-risk populations, compared to the alternative of statin discontinuation. This study found that the use of a pharmacogenomic test is a beneficial strategy for any test costing less than CAN\$356 (Canadian dollars) [35]. This was further supported by another study that found that in highrisk populations, using pharmacogenomic testing is also cost-effective to maintain patients on a statin given the cardiovascular benefit, despite possible risk of rhabdomyolysis [36].

\section{Conclusion}

The results of our study suggest that the discrepancy between statin intolerance in clinical trials and clinical practice cannot be explained by somatosensory amplification, anxiety, depression or pain disorders. The presence of an affective and/or anxiety disorder will likely augment the pain perception and somatization in a SI patient, but the primary cause should be sought elsewhere [8].

Due to the sample size limitation of this study, additional investigations should be performed to fully elucidate the relationship between negative affective syndromes, pain syndromes, pain perception disorders, or somatoform disorders and statin intolerance.

\section{Disclosures}

No disclosures.

\section{Competing interests}

The authors have no competing interests to report.

\section{Funding}

This research did not receive any specific grant from funding agencies in the public, commercial, or not-for-profit sectors.

\section{References}

1. Lloyd-Jones D, Adams RJ, Brown TM, Carnethon M, Dai S, et al. (2010) Executive Summary: Heart Disease and Stroke Statistics-2010 Update. Circulation 121: 948954. [Crossref]

2. Gu Q, Paulose-Ram R, Vicki LB, Kit BK (2014) Prescription Cholesterol-lowering Medication Use in Adults Aged 40 and Over: United States, 2003-2012. NCHS Data Brief 177: 1-8. [Crossref]

3. Bruckert E, Hayem G, Dejager S, Yau C, Bégaud B (2005) Mild to Moderate Muscular Symptoms with High-Dosage Statin Therapy in Hyperlipidemic Patients-The PRIMO Study. Cardiovasc Drugs Ther 19: 403-14. [Crossref]

4. Tobert JA, Newman CB (2015) Statin tolerability: In defence of placebo-controlled trials. Eur J Prev Cardiol 23: 891-896. [Crossref]

5. Banach M, Rizzo M, Toth PP, Farnier M, Davidson MH, et al. (2015) Statin intolerance - an attempt at a unified definition. Position paper from an International Lipid Expert Panel. Arch Med Sci 1: 1-23. [Crossref]

6. Collins R, Armitage J, Parish S, Sleigh P, Peto R (2003) MRC/BHF Heart Protection Study of cholesterol-lowering with simvastatin in 5963 people with diabetes: A randomised placebo-controlled trial. Lancet 361: 2005-2016. [Crossref]

7. Suls J, Martin R (2011) Heart disease occurs in a biological, psychological, and social matrix: Cardiac risk factors, symptom presentation, and recovery as illustrative examples. Ann Behav Med 41: 164-173. [Crossref]

8. Keogh E, Cochrane M (2002) Anxiety sensitivity, cognitive biases, and the experience of pain. J Pain 3: 320-329. [Crossref]

9. Zincir SB, Sunbul M, Aydin Sunbul E, Dalkilic B, Cengiz F, et al.(2014) Evaluation of Alexithymia, Somatosensory Sensitivity, and Health Anxiety Levels in Patients with Noncardiac Chest Pain. Biomed Res Int 2014. 1-6. [Crossref]

10. Dragos D, Tanasescu M (2009) The critical role of psychosomatics in promoting a new perspective upon health and disease. J Med Life 2: 343-349. [Crossref]

11. DiMatteo MR, Lepper HS, Croghan TW (2000) Depression Is a Risk Factor for Noncompliance With Medical Treatment. Arch Intern Med 160: 2101. [Crossref]

12. Yavuz BG, Aydinlar EI, Dikmen PP, Incesu C (2013) Association between somatic amplification, anxiety, depression, stress and migraine. J Headache Pain 14: 2-7. [Crossref]

13. Lovibond PF, Lovibond SH (1995) The structure of negative emotional states: Comparison of the Depression Anxiety Stress Scales (DASS) with the Beck Depression and Anxiety Inventories. Behav Res Ther 33: 335-343. [Crossref]

14. Barsky AJ, Wyshak G, Klerman GL (1990) The Somatosensory Amplification Scale and its relationship to hypochondriasis. J Psychiatr Res 24: 323-334. [Crossref]

15. Melzack R (1987) The short-form McGill pain questionnaire. Pain 30: 191-197.

16. Katon W, Lin EHB, Kroenke K (2007) The association of depression and anxiety with medical symptom burden in patients with chronic medical illness. Gen Hosp Psychiatry 29: 147-155. [Crossref]

17. Russo J, Katon W, Sullivan M, Clark M, Buchwald D (1994) Severity of Somatization and its Relationship to Psychiatric Disorders and Personality. Psychosomatics 35: 546-556.

18. Katon W, Sullivan M, Walker E (2001) Medical Symptoms without Identified Pathology: Relationship to Psychiatric Disorders, Childhood and Adult Trauma, and Personality Traits. Ann Intern Med 134: 917-925. [Crossref]

19. Katon WJ(2003) Clinical and health services relationships between major depression, depressive symptoms, and general medical illness. Biol Psychiatry 54: 216-226. [Crossref]

20. Mueller J, Alpers GW (2016) Two facets of being bothered by bodily sensations: anxiety sensitivity and alexithymia in psychosomatic patients. Compr Psychiatry 47: 489-495. [Crossref]

21. Nakao M, Barsky AJ, Kumano H, Kuboki T (2002) Relationship Between Somatosensory Amplification and Alexithymia in a Japanese Psychosomatic Clinic. Psychosomatics 43: 55-60.

22. Carta MG, Sancassiani F, Pippia V, Bhat KM, Sardu C, et al.(2013) Alexithymia Is Associated with Delayed Treatment Seeking in Acute Myocardial Infarction. Psychother Psychosom 82: 190-192. [Crossref]

23. Rief W, Shaw R, Fichter MM (1998) Elevated Levels of Psychophysiological Arousa and Cortisol in Patients With Somatization Syndrome. Psychosom Med 60: 198-203.

24. White KS, McDonnell CJ, Gervino EV (2011) Alexithymia and anxiety sensitivity in patients with non-cardiac chest pain. $J$ Behav Ther Exp Psychiatry 42: 432-439. [Crossref] 
25. Korhonen M, Pentti MJ, Hartikainen J, Kivimäki M, Vahtera J (2016) Somatic symptoms of anxiety and nonadherence to statin therapy. Int J Cardiol 214: 493-499.

26. Khan S, Holbrook A, Shah BR (2018) Does Googling lead to statin intolerance? Int J Cardiol 262: 25-27. [Crossref]

27. Tobert JA, Newman CB (2016) The nocebo effect in the context of statin intolerance. J Clin Lipidol 10:739-747.

28. Riaz H, Khan AR, Khan MS, Rehman KA, Alansari SAR, et al. (2017) Meta-analysis of Placebo-Controlled Randomized Controlled Trials on the Prevalence of Statin Intolerance. Am J Cardiol 120: 774-781. [Crossref]

29. Gluba-Brzozka A, Franczyk B, Toth PP, Rysz J, Banach M (2016) Molecular mechanisms of statin intolerance. Arch Med Sci 12: 645-658.

30. Patel J, Superko HR, Martin SS, Blumenthal RS, Christopher-Stine L (2015) Genetic and immunologic susceptibility to statin-related myopathy. Atherosclerosis 240: 260271. [Crossref]

31. Siddiqui MK, Maroteau C, Veluchamy A, Tornio A, Tavendale R, et al. (2017) A common missense variant of LILRB5 is associated with statin intolerance and myalgia. Eur Heart J 38: 3569-3575.
32. Musset L, Allenbach Y, Benveniste O, Boyer O, Bossuyt X, et al. (2016) Anti-HMGCR antibodies as a biomarker for immune-mediated necrotizing myopathies: A history of statins and experience from a large international multi-center study. Autoimmun Rev 15: 983-993. [Crossref]

33. Shovman O, Gilburd B, Chayat C, Lazar AD, Amital H, et al. (2017) Anti-HMGCR antibodies demonstrate high diagnostic value in the diagnosis of immune-mediated necrotizing myopathy following statin exposure. Immunol Res 65:276-281.

34. Alvarado-Cardenas M, Marin-Sánchez A, Martínez MA, Martínez-Martínez L, PinalFernandez I, et al. (2016) Statin-associated autoimmune myopathy: A distinct new IFL pattern can increase the rate of HMGCR antibody detection by clinical laboratories. Autoimmun Rev 15: 1161-1166. [Crossref]

35. Mitchell D, Guertin JR, Iliza AC, Fanton-Aita F, LeLorier J (2017) Economic Evaluation of a Pharmacogenomics Test for Statin-Induced Myopathy in Cardiovascular HighRisk Patients Initiating a Statin. Mol Diagnosis Ther 21: 95-105.

36. Mitchell D, Guertin JR, Dubois A, Dubé MP, Tardif JC, et al. (2018) A Discrete Event Simulation Model to Assess the Economic Value of a Hypothetical Pharmacogenomics Test for Statin-Induced Myopathy in Patients Initiating a Statin in Secondary Cardiovascular Prevention. Mol Diagn Ther 22: 241-254. [Crossref]

Copyright: (2019 Singh J. This is an open-access article distributed under the terms of the Creative Commons Attribution License, which permits unrestricted use, distribution, and reproduction in any medium, provided the original author and source are credited. 